\title{
Edge Detection Free Postprocessing for Pseudospectral Approximations*
}

\author{
Scott A. Sarra ${ }^{\dagger}$
}

March 4, 2009

\begin{abstract}
Pseudospectral Methods based on global polynomial approximation yield exponential accuracy when the underlying function is analytic. The presence of discontinuities destroys the extreme accuracy of the methods and the well-known Gibbs phenomenon appears. Several types of postprocessing methods have been developed to lessen the effects of the Gibbs phenomenon or even to restore spectral accuracy. The most powerful of the methods require that the locations of the discontinuities be precisely known. In this work we discuss postprocessing algorithms that are applicable when it is impractical, or difficult, or undesirable to pinpoint all discontinuity locations.
\end{abstract}

keywords: Fourier, Chebyshev, Pseudospectral, Gibbs, Spectral filtering, Digital Total Variation filtering.

\section{Introduction}

Pseudospectral methods are based on global, interpolating, orthogonal polynomial expansions of the form

$$
\mathcal{I}_{N} f(x)=\sum_{k} a_{k} \phi_{k}(x)
$$

The expansion coefficients are denoted by $a_{k}$ and the basis functions $\phi_{k}(x)$ can be standardized to the interval $\Omega=[-1,1]$. The expansion satisfies

\footnotetext{
${ }^{*}$ This work was partially supported by NSF grant DMS-0609747

${ }^{\dagger}$ Department of Mathematics, Marshall University, One John Marshall Drive, Huntington, WV 25755. e-mail: sarra@marshall.edu
} 
$\mathcal{I}_{N} f\left(x_{i}\right)=f\left(x_{i}\right)$ at $N+1$ interpolation sites $x_{i}$. Interpolation means that $f(x)$, the function that is being approximated, is a known function (at least at the interpolation sites) while the terms collocation and pseudospectral are applied to methods based on the expansion (1) for solving differential equations for an unknown function $f(x)$. We refer to both situations as spectral approximation or spectral methods. Detailed information on spectral methods may be found in the standard references $[1,2,8,18]$. When the underlying function is periodic trigonometric (Fourier) polynomials are employed as basis functions while a popular choice for non-periodic problems are the Chebyshev polynomials. Spectral methods are exponentially accurate when the underlying function is sufficiently smooth. However, the presence of discontinuities in the function or in its derivatives reduces the accuracy of the methods. The reduced accuracy is visible in the form of oscillations that characterize the well-known Gibbs phenomenon which reduces accuracy to first order away from discontinuities and to $\mathcal{O}(1)$ in the neighborhoods of jumps.

A number of methods have been suggested for the purpose of reducing or eliminating the Gibbs phenomenon. They include: spectral filtering, physical space filtering using mollifiers, digital total variation filtering, rational reconstruction, and a variety of direct and indirect reprojection methods. The most powerful methods need to know the exact location of all discontinuities (or edges). Methods to locate edges in spectral data are developed in references $[5,6,7]$. Computational efficiency in higher dimensions is also an issue with some of the postprocessing methods. An overview of spectral postprocessing methods may be found in reference [16].

Edge detection algorithms may have difficulty pinpointing the exact location of discontinuities in several situations that include: the underlying function having jumps of various magnitudes, and discontinuities being present in both the function and its derivative. Additional difficulty is added in two dimensions when the edges are not aligned with the cartesian grid. The cartesian grid problem is discussed further in [4]. When edges are unable to be located precisely many of the postprocessing algorithms are rendered useless or at least suffer a severe loss of accuracy. Methods that require one or more function dependent parameters in each smooth region present additional difficulties, especially in higher dimensions.

In this work we present a computationally efficient edge detection free algorithm that requires only three global parameters. The method combines spectral filtering, which theoretically recovers spectral accuracy at points sufficiently away from discontinuities, with Digital Total Variation (DTV) filtering. Computational evidence indicates that DTV filtering is superior 
to spectral filtering in neighborhoods of discontinuities. A built-in smoothness indicator in the DTV algorithm determines which method is used at each point. In reference [4] a hybrid method was developed that uses the Gegenbauer reconstruction method in neighborhoods of discontinuities and a spectral filter away from the discontinuities. The main purpose of the hybrid Gegenbauer/spectral filter method was to reduce the high computational cost of the Gegenbauer method by using the less computationally expensive spectral filter in regions away from edges. However, the hybrid Gegenbauer/spectral filter method needs to know the exact location of edges to be effective.

Theoretical results support the use of spectral filters for Fourier and Chebyshev methods. Thus we focus on these two methods. There is essentially no theory to support the use of spectral filters in general polynomial expansions beyond Fourier and Chebyshev polynomials. However much computational evidence exists supporting that spectral filters are effective for other polynomial expansions as well. Recently some progress has been made to quantify the effects of spectral filtering on Legendre expansions [9]. The hybrid spectral/DTV filtering algorithm is applicable to any orthogonal polynomial expansion for which the use of spectral filtering is justifiable.

Next we summarize spectral and DTV filtering and then describe the hybrid algorithm and present numerical examples.

\section{Spectral Filters}

Spectral filters [19] lessen the effects of the Gibbs phenomenon by working in transform space as

$$
\mathcal{F}_{N} f(x)=\sum_{k} \sigma(k / N) a_{k} \phi_{k}(x)
$$

A spectral approximation can be postprocessed efficiently by a spectral filter in $\mathcal{O}(N \log N)$ floating point operations using the Fast Fourier Transform. The convergence rate of the filtered approximation is determined solely by the order, $\rho>1$, of the filter and the regularity of the function away from the point of discontinuity. If $\rho$ is chosen increasing with $N$, the filtered expansion recovers exponential accuracy away from a discontinuity. Assuming that $f(x)$ has a discontinuity at $x_{0}$ and setting $d(x)=x-x_{0}$, the estimate

$$
\left|f(x)-\mathcal{F}_{N}(x)\right| \leq \frac{K}{d(x)^{\rho-1} N^{\rho-1}}
$$


holds where $K$ is a constant. If $\rho$ is sufficiently large, and $d(x)$ is not too small, the error goes to zero faster than any finite power of $N$, i.e. spectral accuracy is recovered. When $x$ is close to a discontinuity the error increases. If $d(x)=\mathcal{O}(1 / N)$ then the error estimate is $\mathcal{O}(1)$.

The spectral filter that we have used in the hybrid algorithm is the $\rho^{\text {th }}$ order Vandeven filter

$$
\sigma(\omega)=1-\frac{(2 \rho-1) !}{(p-1) !} \int_{0}^{|\omega|} t^{\rho-1}(1-t)^{\rho-1} d t
$$

which is known to have some optimal properties among all spectral filters [19]. However, other spectral filters could be used in the hybrid method as well.

\section{Digital Total Variation Filtering}

The Total Variation (TV) de-noising model is a popular image processing method to remove noise from a digital image. The model formulates a minimization problem which leads to a nonlinear Euler-Lagrange PDE to be solved by numerical PDE methods. In [3, 13] the authors develop a discrete version of the TV model on a graph and refer to it as Digital Total Variation (DTV) filtering. Viewing an oscillatory function as an image with noise, the DTV method was used to postprocess spectral approximations in [14] and Radial Basis Function approximations in [15]. The method works with point values in physical space and not with the spectral expansion coefficients as the spectral filter does. The DTV method does not need to know the location of edges. The point values may be located at scattered, non-structured sites, in complex geometries. While the method does mitigate the effects of the Gibbs phenomenon it does not make any claims of restoring spectral accuracy.

General points in the computational domain are denoted by $\alpha, \beta, \cdots$. The notation $\alpha \sim \beta$ indicates that $\alpha$ and $\beta$ are neighbors. All the neighbors of a point $\alpha$ are denoted by

$$
N_{\alpha}=\{\beta \in \Omega \mid \beta \sim \alpha\} .
$$

In one dimension, $N_{\alpha}$ consists simply of the points to the left and right of the point being postprocessed. In two space dimensions there is more than one way [16] to define $N_{\alpha}$. In this work we use an eight point neighborhood (figure 1),

$$
N_{\alpha}^{8}=\left\{\alpha_{i, j+1}, \alpha_{i+1, j+1}, \alpha_{i+1, j}, \alpha_{i+1, j-1}, \alpha_{i, j-1}, \alpha_{i-1, j-1}, \alpha_{i-1, j}, \alpha_{i-1, j+1}\right\} .
$$


The graph variational problem is to minimize the fitted TV energy

$$
E_{\lambda}^{T V}(u)=\sum_{\alpha \in \Omega}\left|\nabla_{\alpha} u\right|_{a}+\frac{\lambda}{2} \sum_{\alpha \in \Omega}\left(u_{\alpha}-u_{\alpha}^{0}\right)^{2}
$$

where $u^{0}$ is the spectral approximation containing the Gibbs oscillations and $\lambda$ the user specified fitting parameter. The unique solution to this problem is the solution of the nonlinear restoration equation

$$
\sum_{\beta \sim \alpha}\left(u_{\alpha}-u_{\beta}\right)\left(\frac{1}{\left|\nabla_{\alpha} u\right|_{a}}+\frac{1}{\left|\nabla_{\beta} u\right|_{a}}\right)+\lambda\left(u_{\alpha}-u_{\alpha}^{0}\right)=0
$$

where the regularized location variation or strength function at any point $\alpha$ is defined as

$$
\left|\nabla_{\alpha} u\right|_{a}=\left[\sum_{\beta \in N_{\alpha}}\left(u_{\beta}-u_{\alpha}\right)^{2}+a^{2}\right]^{1 / 2} .
$$

The regularization parameter $a$ is a small (we have used $a=0.0001$ in the numerical examples) value used to prevent a zero local variation and division by zero.

To solve the nonlinear system, time marching with the explicit Euler method is used to advance a preconditioned form of the (7)

$$
\frac{d u_{\alpha}}{d t}=\sum_{\beta \sim \alpha}\left(u_{\alpha}-u_{\beta}\right)\left(1+\frac{\left|\nabla_{\alpha} u\right|_{a}}{\left|\nabla_{\beta} u\right|_{a}}\right)+\lambda\left|\nabla_{\alpha} u\right|_{a}\left(u_{\alpha}-u_{\alpha}^{0}\right)
$$

to a steady state. Typically less 100 time steps are required. An effective stopping criteria for the time marching is for the relative $L^{1}$ residual between two consecutive time steps to be less than some tolerance, i.e.,

$$
\frac{\left\|u^{[k+1]}-u^{[k]}\right\|_{L^{1}}}{\left\|u^{[k]}\right\|_{L^{1}}} \leq \text { tol. }
$$

Equation (9) is essentially a local finite difference approximation with each time iteration taking $\mathcal{O}(N)$ floating point operations. Thus, the DTV filtering algorithm is computationally efficient as long as not too many time marching steps are taken.

An optimal value of the fitting parameter is not known. However, a large range of values for the fitting parameter results in a "good" postprocessing. In general, stronger oscillations are best handled with a small fitting parameter $(<10)$ while weaker oscillations require a larger value of the fitting parameter. More details on selecting the value of the fitting parameter can be found in reference [14]. 


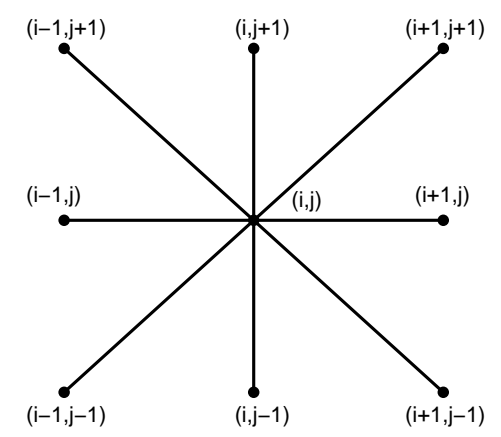

Figure 1: 2d DTV neighborhood $N_{\alpha}^{8}$

\section{Hybrid DTV-Spectral Filter}

In the hybrid DTV-Spectral filter method, the initial evaluation of the strength function (8) from the DTV method is normalized

$$
\mathcal{S}=\frac{\left|\nabla_{\alpha} u\right|_{a}}{\max \left|\nabla_{\alpha} u\right|_{a}}
$$

and used to determine which method is applied. If $\mathcal{S}[f(x)]<\mathcal{S}_{\max }$ the spectral filter is applied at $x$. Otherwise the DTV postprocessed approximation is used. This allows the DTV filter to be applied in the neighborhood of discontinuities where the spectral filter is not as effective. Around discontinuities a strong spectral (small $\rho$ ) filter rounds off sharp corners while a weaker filter does not remove the Gibbs oscillations. The spectral filter is applied away from discontinuities where it can restore spectral accuracy. Three global parameters must be specified in the hybrid method: the spectral filter order $\rho$, the DTV fitting parameter $\lambda$, and $\mathcal{S}_{\max }$. The parameters $\rho$ and $\lambda$ should be specified just as they are in the non-hybrid application of the filters while computational evidence indicates that setting $\mathcal{S}_{\max }$ in the range $0.005 \leq \mathcal{S}_{\max } \leq 0.035$ leads to good results. Due to the global nature of the DTV method the entire solution must be DTV postprocessed. Then, based on the DTV strength function, the spectral filter only needs to be computed at the points where the DTV filtered solution is not used.

\section{$5 \quad$ Numerical Examples}

All example functions and PDE solutions are benchmark examples from the Matlab Postprocessing Toolbox [16]. The first example is a one-dimensional 

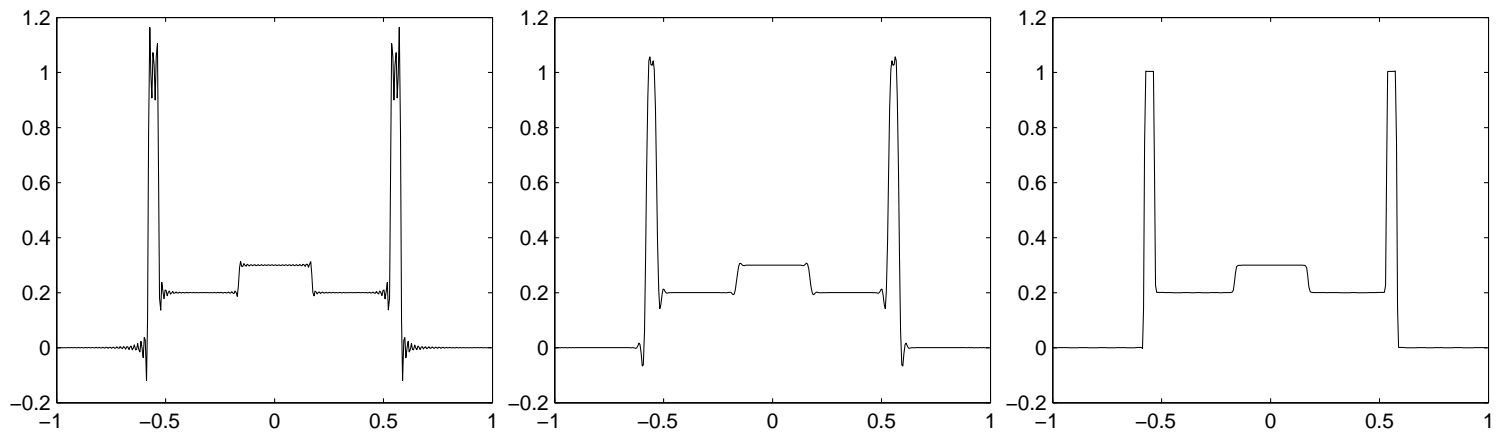

Figure 2: Left: Fourier approximation. Middle: spectral filter postprocessed, $\rho=10$. Right: DTV filtered postprocessed, 200 time steps with $\lambda=15$.

slice of the modified Shepp-Logan brain phantom from the Matlab Image Processing Toolbox. In the left image of figure 2 the oscillatory $N=250$ Fourier approximation is evaluated at $M=398$ evenly spaced points. The example has jumps of various magnitudes as well as jumps that are close together. Both situations make it difficult for edge detection algorithms to accurately locate all the edges.

The spectral filtered and DTV filtered approximations are shown in figure 2 and the errors in figure 4. The results are typical of both methods. The weak spectral filter greatly improves accuracy away from the edges but leaves oscillations around the discontinuities. A stronger filter would have rounded off the sharp corners at the discontinuities. The DTV filter does better around the jumps but does not recover as much accuracy away from the edges as the spectral filter does. In the hybrid method, the normalized DTV strength function (right image of figure 3) is used to determine where each filter is applied. At points where $\mathcal{S}[f(x)]<\mathcal{S}_{\max }=0.005$ the spectral filter is applied. Otherwise the DTV postprocessed approximation is used. The postprocessing took 0.09 seconds using the algorithm implemented in Matlab running on a desktop computer that is representative of current computer technology.

It is inevitable that the hybrid postprocessed solution has small discontinuities at transition points between postprocessing methods. Blending schemes could be implemented to ease the transitional discontinuities. One approach would be to simply average the spectral filtered and DTV postprocessed solutions at points around transitions where the normalized strength function $\mathcal{S}$ is such that $\mathcal{S}_{1} \leq \mathcal{S} \leq \mathcal{S}_{2}$ where $\mathcal{S}_{1} \leq \mathcal{S}_{\max } \leq \mathcal{S}_{2}$. The param- 

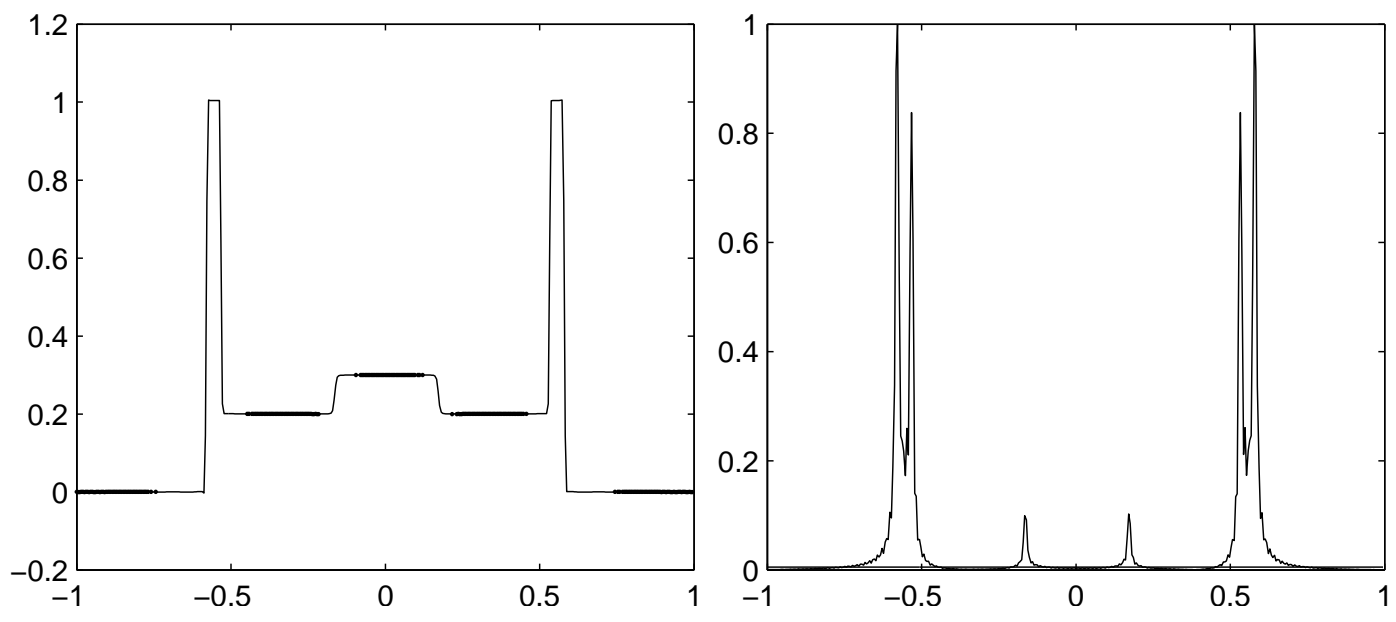

Figure 3: Left: Hybrid spectral/DTV filter with $\mathcal{S}_{\max }=0.005$. The darker segments of the graph indicate where spectral filtering has been applied. Right: normalized DTV strength function (11)

eters $\mathcal{S}_{1}$ and $\mathcal{S}_{2}$ would be user specified and problem dependent. However, the requirement of additional user defined parameters seems to take away from the main strength of the hybrid method. Unless the small transitional jumps are unacceptable in applications, it seems best to accept the small transitional jumps in order to maintain the simplicity and ease of application of the hybrid method.

The next two examples involve the Chebyshev Pseudospectral method for PDEs. The physical space grid point locations are the non-uniformly spaced points [11]

$$
x_{j}=\frac{\arcsin [-\gamma \cos (\pi j / N)]}{\arcsin (\gamma)}, \quad j=0,1, \ldots N, \quad 0<\gamma<1 .
$$

The first example is the Chebyshev pseudospectral approximation of the linear advection equation

$$
\frac{\partial u}{\partial t}+\frac{\partial u}{\partial x}=0
$$

on a grid determined by $N=256$ and $\gamma=0.99$ in (12). A piecewise constant initial condition is advanced to time $T=2$ with inflow-outflow boundary conditions applied and with a fourth-order Runge-Kutta method using $\Delta t=0.002$ (left image of figure 5). The hybrid DTV/spectral filtered approximation is shown in the left image (and postprocessed error in the 

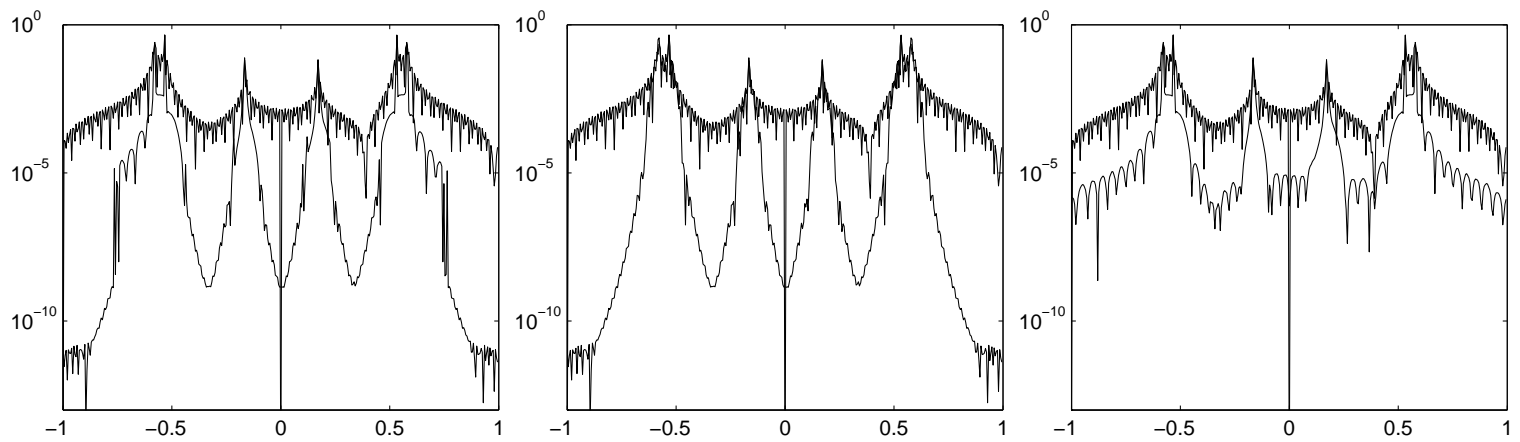

Figure 4: Left: hybrid error. Middle: spectral filter error. Right: DTV error.

right image) of figure 6 with the graph of the postprocessed solution being thicker where the spectral filter has been applied. The spectral filter is of order $\rho=10$. The DTV filter with $\lambda=20$ was applied at points where $\mathcal{S}[u(x)]<\mathcal{S}_{\max }=0.01$.

The second Chebyshev example uses a Chebyshev Super Spectral Viscosity method on a grid determined by $N=512$ and $\gamma=0.999$ on the interval $[-1,1]$ to approximate the density variable of Sod's problem [17] for the Euler equations of gas dynamics. The addition of a very weak spectral viscosity is necessary to stabilize the Chebyshev pseudospectral method when it is applied to nonlinear hyperbolic conservation laws. The particular super spectral viscosity operator [12] used to stabilize the method is

$$
\left(C N^{1-2 s}\right)(-1)^{s+1}\left[\sqrt{1-x^{2}} \frac{\partial}{\partial x}\right]^{2 s} \mathcal{I}_{N} u
$$

with $C=1$ and $s=5$. The solution (right image of figure 5) is advanced to to time $T=2$ using $\Delta t=0.002$ with a fourth-order Runge-Kutta method. The spectral viscosity method can be interpreted as the application of a very weak spectral filter. In this case, a second application of a spectral filter is unable to improve on the DTV filter in any region of the domain and the hybrid method has no advantage over the DTV method. The DTV postprocessed solution, using 200 time steps and $\lambda=100$, and error are shown in figure 7 . The postprocessing took 0.06 seconds of computer time.

The performance of the DTV method near the contact discontinuity at $x=0$ is rather unimpressive. However in this example, as well as in other numerical experiments, DTV filtering does produce a more accurate postprocessed solution than does spectral filtering near discontinuities in the 

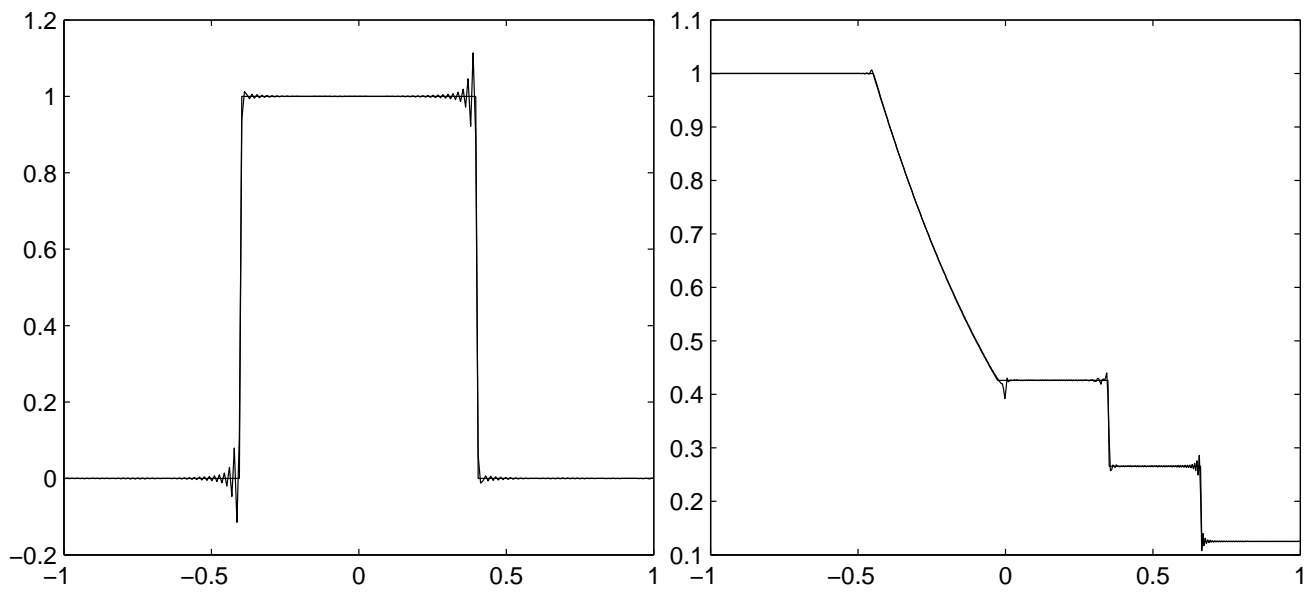

Figure 5: Left: Chebyshev pseudospectral approximation of the linear advection equation, $N=256$. Right: Chebyshev Super Spectral Viscosity approximation of the density variable from Sod's problem for the Euler equations of gas dynamics, $N=512$.

derivative.

To illustrate the effects of the fitting parameter $\lambda$, and that a large range of fitting parameters produce good results, we perform the postprocessing again with $\lambda=10$ (upper images of figure 8) and $\lambda=300$ (lower images of figure 8) rather than $\lambda=100$. Using $\lambda=300$ results in some small oscillations remaining in the immediate location of discontinuities in the postprocessed solution. With $\lambda=10$ some smearing of sharp edges is noticeable in the postprocessed solution. Even at these two largely differing values of the fitting parameter the two postprocessed solutions are remarkably similar except for right at the discontinuities. Additionally, a large range of fitting parameter around $\lambda=100$, for example $20 \leq \lambda \leq 180$, will result in a postprocessed solution that is visibly free of both oscillatory and smearing effects around discontinuities.

The first $2 \mathrm{~d}$ example is the periodic piecewise defined function

$$
f(x, y)=a(x, y)-b(x, y)
$$

where

$$
a(x, y)= \begin{cases}\sin [\pi(x+y)] & |x|+|y| \leq 0.5 \\ 0 & \text { otherwise }\end{cases}
$$



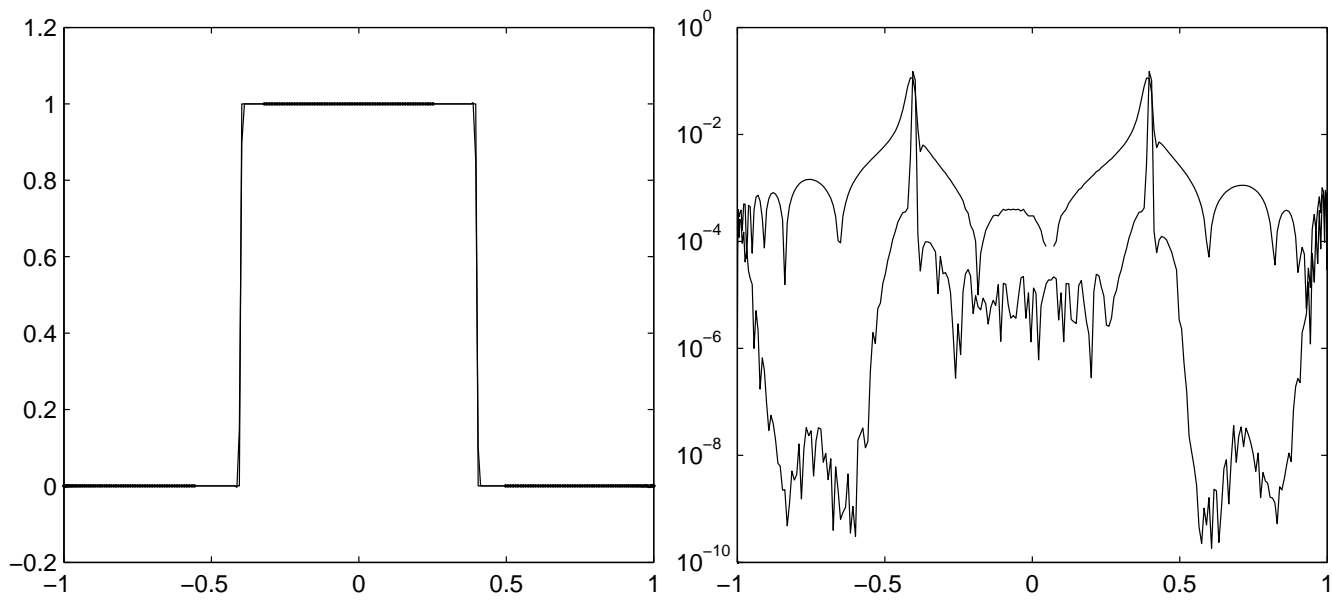

Figure 6: Left: Hybrid spectral-DTV postprocessed Chebyshev pseudospectral approximation. Right: Hybrid postprocessing error (lower) vs. Chebyshev pseudospectral error.

and

$$
b(x, y)= \begin{cases}\sin [\pi(x+y)] & |x|+|y|>0.75 \\ 0 & \text { otherwise }\end{cases}
$$

A contour plot of function (13) is shown in the upper left image of figure 9 and its Fourier approximation in the upper right image of the figure. The function features discontinuities that are not orthogonal to the cartesian grid and edge detection algorithms may have difficulty pinpointing the exact location of the edges. The function has many subintervals of smoothness of various sizes. Postprocessing methods, such as the Gegenbauer reprojection method, that require two function dependent parameters in each subinterval would be difficult to accurately apply to this example. The hybrid postprocessed approximation is shown in the lower left image of figure 9. The DTV normalized strength function which indicates which method of the hybrid method is applied is in the lower right image of the figure 9 .

The final example is the full two-dimensional modified Shepp-Logan brain phantom, that we considered a one-dimensional slice of in a previous example. The results of applying the hybrid algorithm to the phantom are summarized in figure 10. A close-up of a small circle and a small circle intersecting a larger circle is in figure 11. The blown-up region would be challenging for edge detection algorithms. Postprocessing at the 250,000 points of the image took less than 10 seconds. 

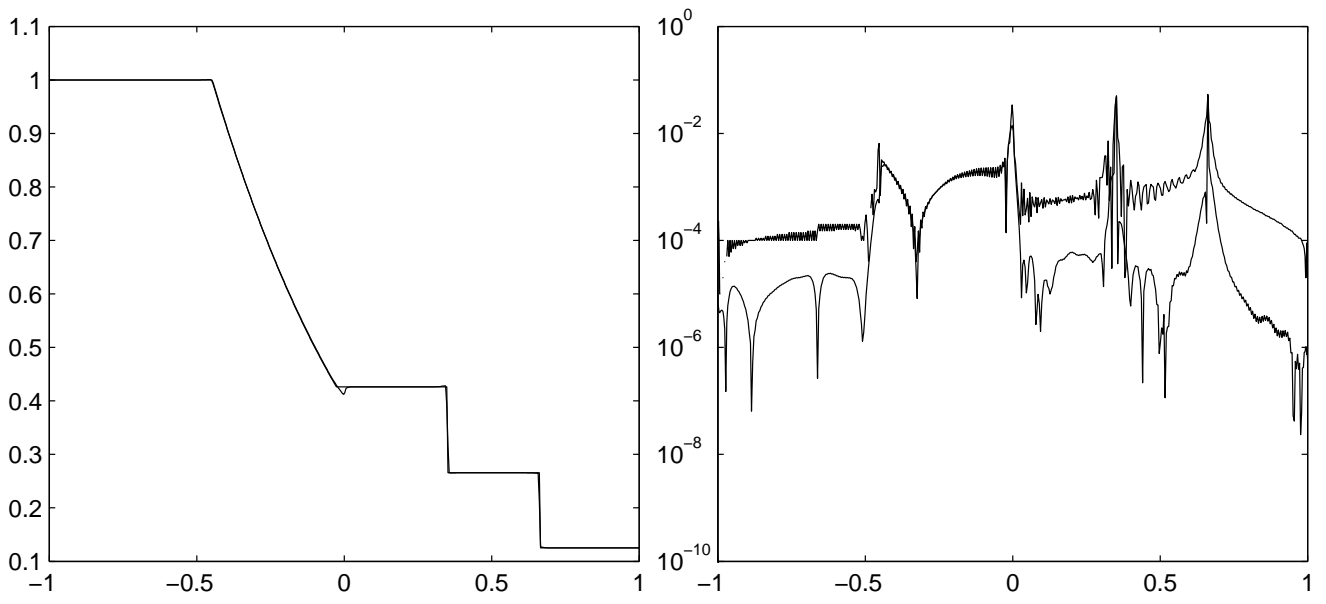

Figure 7: Left: DTV postprocessed Chebyshev super spectral viscosity approximation. Right: DTV postprocessing error (lower) vs. Chebyshev pseudospectral error.

The DTV filter sharply resolves discontinuities while the spectral filter recovers spectral accuracy at points sufficiently away from the discontinuities. The hybrid DTV/Spectral filter results can be compared to other attempts to postprocess the brain phantom. A hybrid Gegenbauer reprojection/spectral filter method, which requires that the exact locations of all discontinuities be pinpointed, was applied in [4]. The Fourier representation of the brain phantom was postprocessed in [10] using the Inverse Polynomial Reprojection method that needs the locations of the edges to be known precisely. However, the authors state that "The edge locations are assumed as known quantities in this work so that the edge effect is minimized". The results in [10] illustrate how powerful many of the methods are that require the exact location of discontinuities. However, locating the exact location of all edges in a complicated two-dimensional function is non-trivial. Since the hybrid DTV/spectral filter does not require that edge locations be known, the method is very attractive for this type of problem.

\section{Summary}

The hybrid DTV/spectral filter combines the best of two computationally efficient edge detection free postprocessing methods for spectral approximations. One method works in transform space with the spectral coefficients 
while the other method work in physical space with point values. The hybrid algorithm uses the DTV strength function to determine whether the spectral or DTV filters are used as the postprocessing method. The hybrid algorithm is more accurate away from discontinuities, than is DTV filtering alone, and is more accurate than spectral filtering alone in the neighborhoods of discontinuities. Spectral viscosity methods, in which a weak spectral filter has been applied to stabilize the pseudospectral methods are an exception. Further spectral filtering is usually unable to recover more accuracy than the DTV filter alone.

In one dimension, the hybrid algorithm is well suited for use in situations where edge detection methods have difficulty. This is the case when the underlying function has jumps of various magnitudes or has jumps in both the function and its derivative. Additional difficulties may be encountered in two dimensions if discontinuities are not orthogonal to the cartesian grid. Our numerical examples indicated that the hybrid methods performs well in this case also. Many postprocessing methods that use edge detection also require that function dependent parameters must be specified in each smooth subregion. In practice, it proves virtually impossible to get an accurate postprocessing of a complicated function when so many parameters must be chosen. The hybrid spectral-DTV filter needs only three global parameters. Since the spectral filter is only activated away from discontinuities, a large range of filter orders $\rho$ give good results. The choice of $\rho$ should be coordinated with $\mathcal{S}_{\max }$, as for fixed $N$, a stronger spectral filter will have a more noticeable effect around a discontinuity that a weaker filter. A large range of values for the fitting parameter $\lambda$ produces good results. The lack of critical dependence on only a small number of global parameters makes the hybrid spectral-DTV filter a near black box algorithm for mitigating the effects of the Gibbs Phenomenon in spectral approximations.

\section{References}

[1] J. P. Boyd. Chebyshev and Fourier Spectral Methods. Dover, second edition, 2000. 1

[2] C. Canuto, M. Hussaini, A. Quarteroni, and T. Zang. Spectral Methods: Fundamentals in Single Domains. Springer, 2006. 1

[3] T. Chan, S. Osher, and J. Shen. The digital TV filter and nonlinear denoising. IEEE Transactions on Image Processing, 10(2), 2001. 3 
[4] A. Gelb. A hybrid approach to spectral reconstruction of piecewise smooth functions. Journal of Scientific Computing, 15:293-322, 2001. 1,5

[5] A. Gelb and D. Cates. Detection of edges in spectral data III: Refinement of the concentration method. To appear in Journal of Scientific Computing, 2008. 1

[6] A. Gelb and E. Tadmor. Detection of edges in spectral data. Applied and Computational Harmonic Analysis, 7:101-135, 1999. 1

[7] A. Gelb and E. Tadmor. Detection of edges in spectral data II: Nonlinear enhancement. SIAM Journal on Numerical Analysis, 38(4):13891408, 2000. 1

[8] J. Hesthaven, S. Gottlieb, and D. Gottlieb. Spectral Methods for TimeDependent Problems. Cambridge University Press, 2007. 1

[9] J. S. Hesthaven and R. M. Kirby. Filtering in Legendre spectral methods. To appear Mathematics of Computation, 2008. 1

[10] J.-H. Jung and B. Shizgal. Inverse polynomial reconstruction of two dimensional Fourier images. Journal of Scientific Computing, 25:367$399,2005.5$

[11] R. Kosloff and H. Tal-Ezer. A modified Chebyshev pseudospectral method with an $\mathrm{O}(1 / n)$ time step restriction. Journal of Computational Physics, 104:457-469, 1993. 5

[12] H. Ma. Chebyshev-Legendre super spectral viscosity method for nonlinear conservation laws. SIAM J. Numer. Anal, 35:893-908, 1998. 5

[13] S. Osher and J. Shen. Digitized PDE method for data restoration. In G. Anastassiou, editor, Analytic-Computational Methods in Applied Mathematics, chapter 16, pages 751-771. Chapman and Hall/CRC, 2000. 3

[14] S. A. Sarra. Digital Total Variation filtering as postprocessing for Chebyshev pseudospectral methods for conservation laws. Numerical Algorithms, 41:17-33, 2006. 3, 3

[15] S. A. Sarra. Digital Total Variation filtering as postprocessing for Radial Basis Function Approximation Methods. Computers and Mathematics with Applications, 52:1119-1130, 2006. 3 
[16] S. A. Sarra. The Matlab postprocessing toolkit. Submitted to ACM Transactions on Mathematical Software, 2008. 1, 3, 5

[17] G. Sod. A survey of several finite difference methods for systems of nonlinear hyperbolic conservation laws. Journal of Computational Physics, $27: 1-31,1978.5$

[18] L. N. Trefethen. Spectral Methods in Matlab. SIAM, Philadelphia, 2000. 1

[19] H. Vandeven. Family of spectral filters for discontinuous problems. SIAM Journal of Scientific Computing, 6:159-192, 1991. 2, 2 

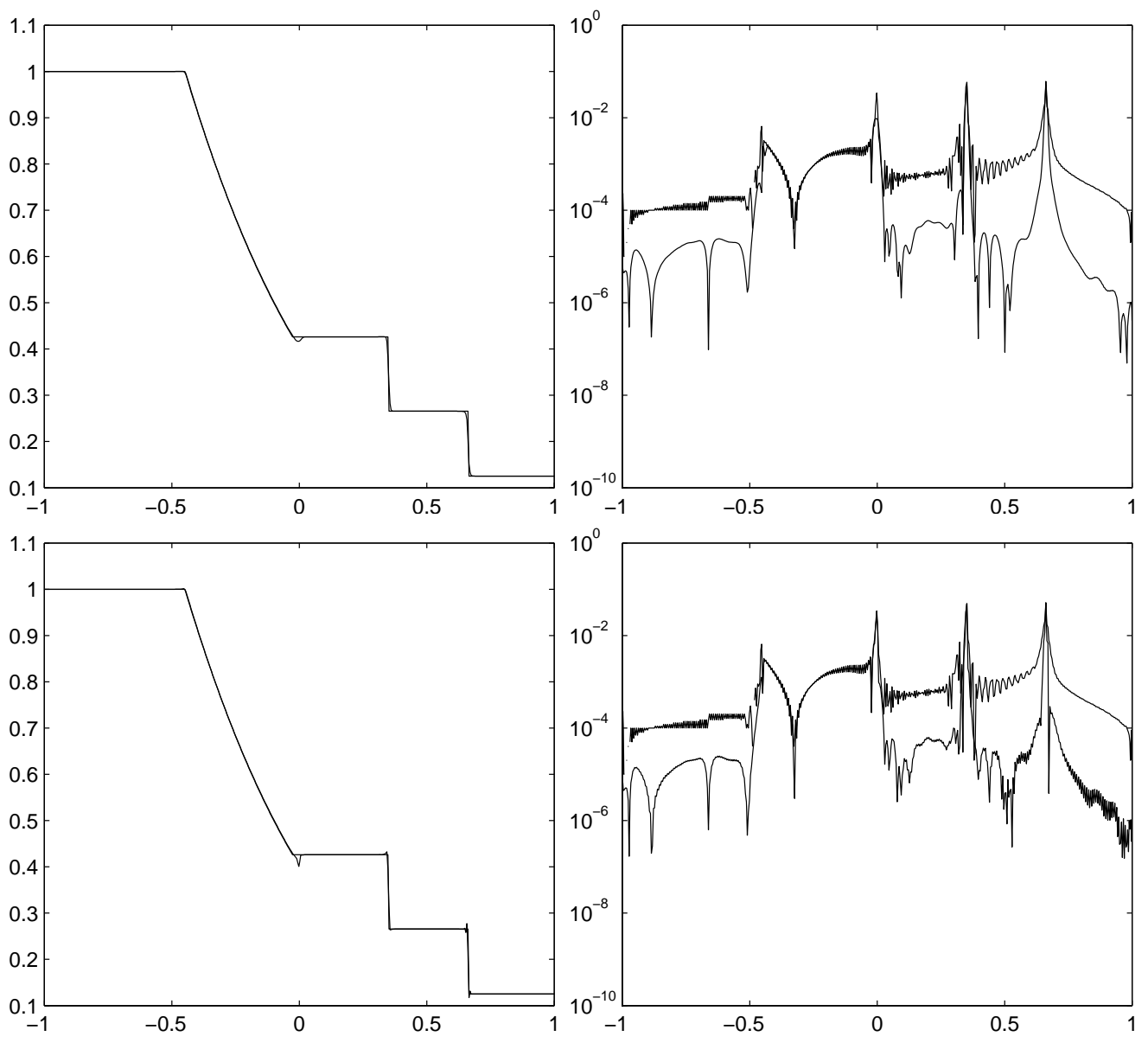

Figure 8: Upper: $\lambda=10$. Lower: $\lambda=300$. Left: DTV postprocessed Chebyshev super spectral viscosity approximation vs. exact. Right: DTV postprocessing error (lower curve) vs. Chebyshev pseudospectral error. 

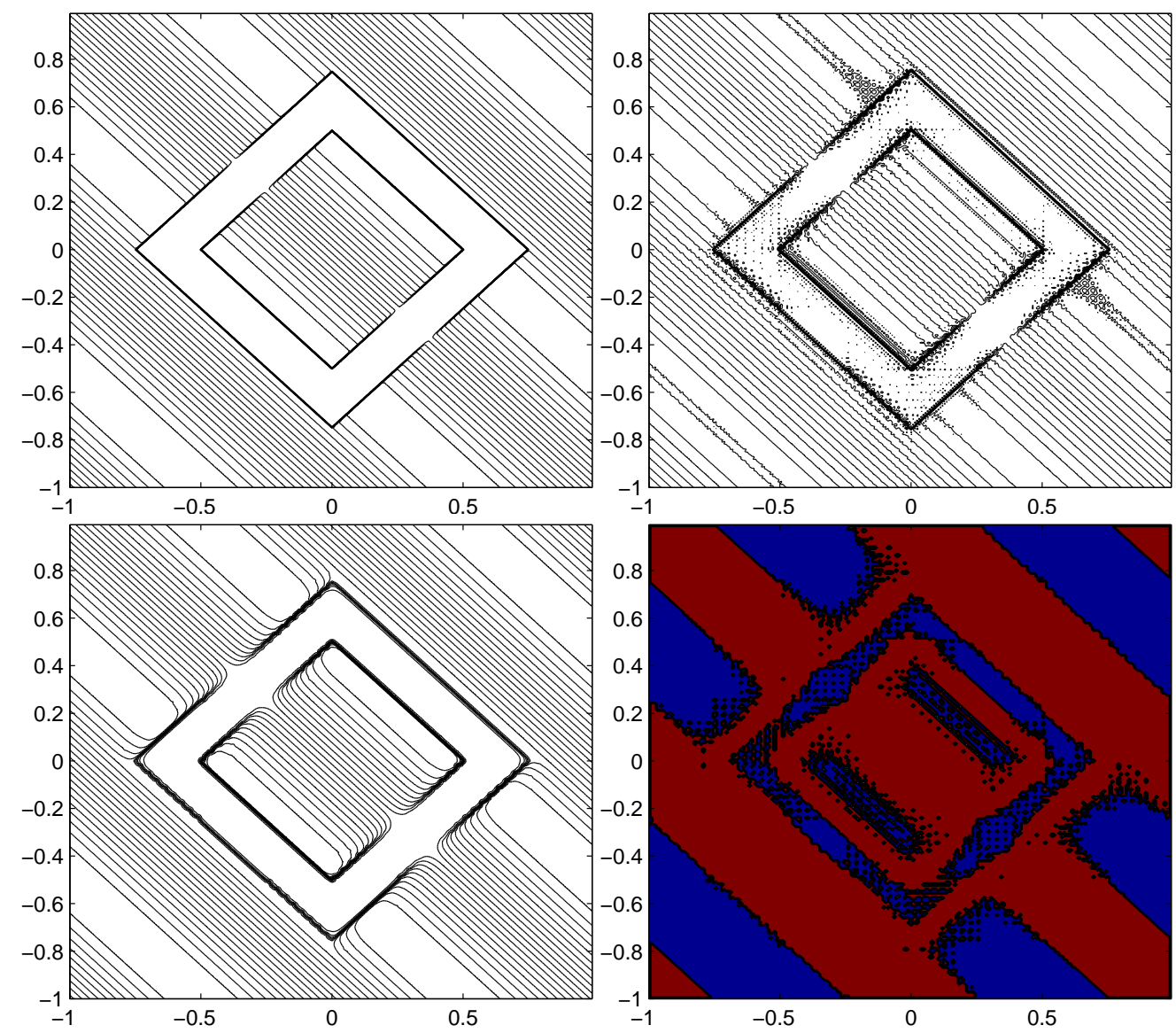

Figure 9: Upper left: function (13). Upper right: Fourier approximation of function (13), $192 \times 192$ Fourier approximation evaluated on a $250 \times 250$ uniform grid. Lower left: Hybrid postprocessed, $\rho=8, \lambda=8$, and $\mathcal{S}_{\max }=$ 0.03. Lower right: DTV filter application area (red), spectral filter (blue). 

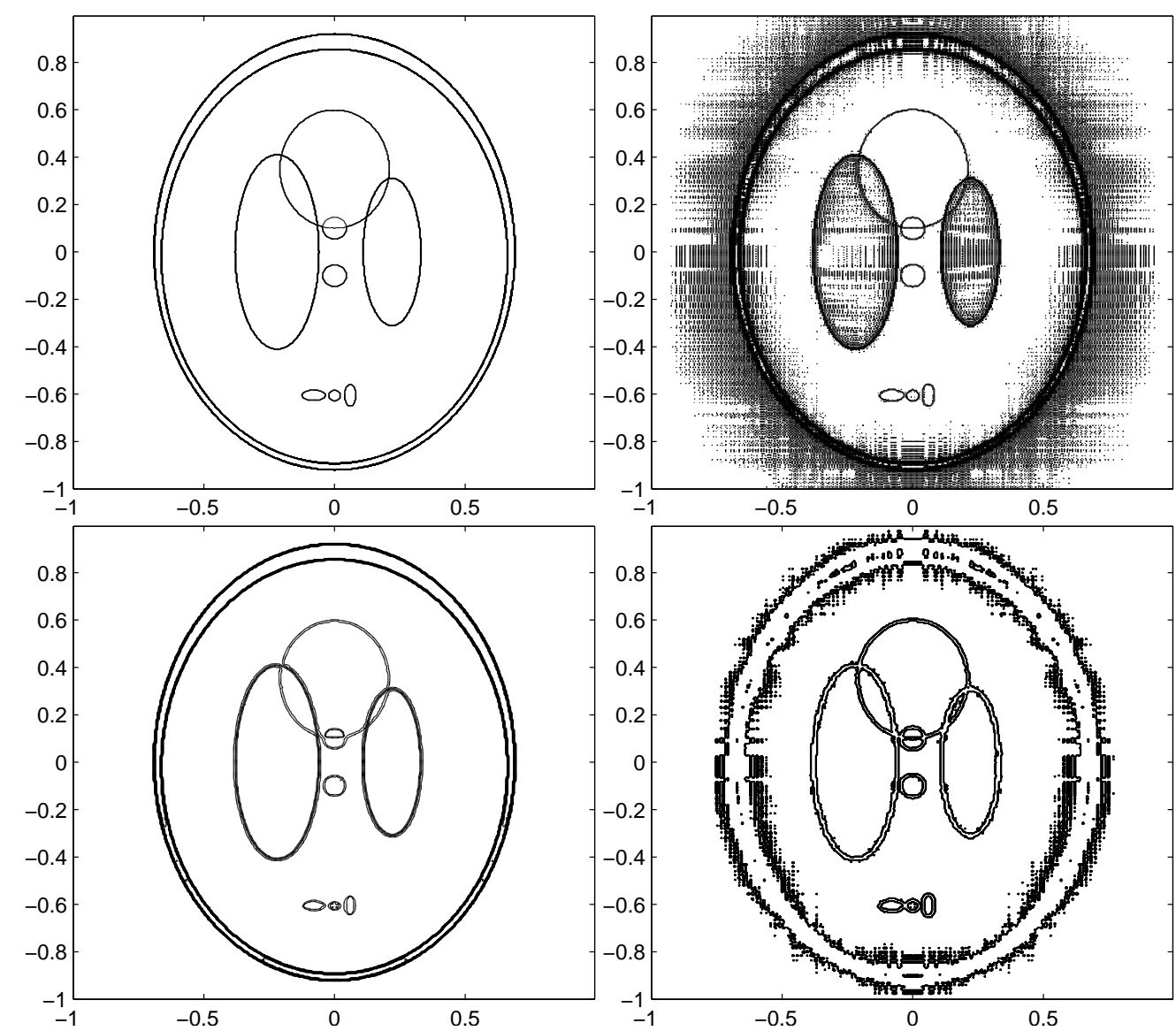

Figure 10: Upper left: $500 \times 500$ contour plot of the modified Shepp-Logan brain phantom. Upper right: $384 \times 384$ Fourier approximation evaluated on a $500 \times 500$ uniform grid. Lower left: Hybrid postprocessed, $\rho=2, \lambda=12$, and $\mathcal{S}_{\max }=0.035$. Lower right: DTV filter application area (black), spectral filter (white). 


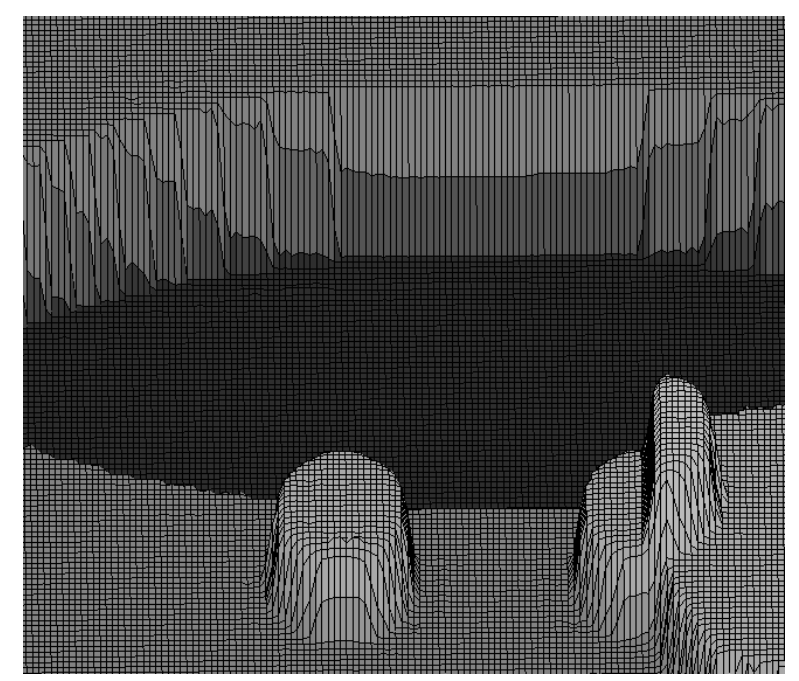

Figure 11: Close up surface plot of a region of detail centered around the point $(0,0.1)$ of the hybrid postprocessed approximation from the lower left image of figure 10 